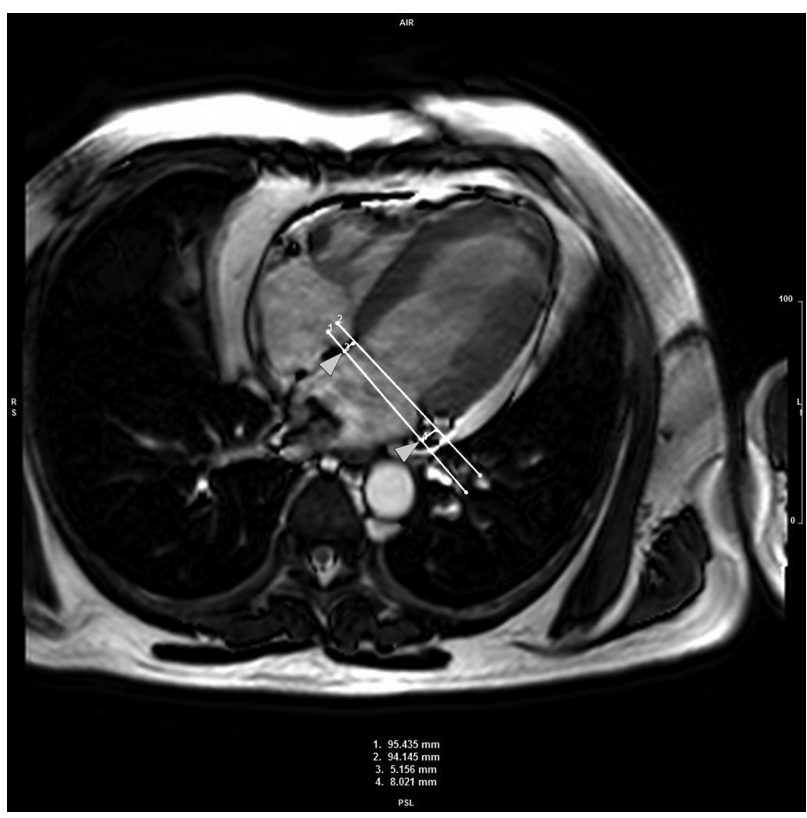

Abstract 16 Figure 1 MAPSE measurements were taken in the fourchamber cine.

\section{ROUTINE CARDIAC MRI SCREENING REVEALS REDUCED PREVALENCE OF STRUCTURAL CARDIOVASCULAR ABNORMALITIES IN PATIENTS WITH TURNER SYNDROME}

\begin{abstract}
${ }^{1}$ Agnes Hamilton-Baillie, ${ }^{1,2}$ Christopher M Jones, ${ }^{1}$ Caroline Packer, ${ }^{2}$ Andy Toogood, ${ }^{2}$ Paul Clift. 'College of Medical and Dental Sciences, University of Birmingham, UK; ${ }^{2}$ University Hospitals Birmingham NHS Foundation Trust, Birmingham, UK
\end{abstract}

\subsection{6/heartjnl-2015-307845.17}

Background Turner syndrome has been associated with significant rates of cardiovascular anomalies. Current guidelines for the management of this condition suggest evaluation of the aorta via cardiac magnetic resonance (CMR) imaging or echocardiography every 5 to 10 years to evaluate each patient's potential for aortic dissection, without prior risk stratification. The current spectrum and frequency of structural cardiovascular anomalies in Turner syndrome is however unclear, with much of the current literature based on historical cohorts.

Purpose To determine through CMR screening the current prevalence of structural cardiovascular anomalies amongst patients with Turner syndrome.

Methods We retrospectively reviewed the medical records of adult patients diagnosed with Turner syndrome who had been seen within a large NHS tertiary referral centre and who had undergone routine surveillance for structural cardiovascular anomalies. Each underwent CMR imaging. Data relating to patient demographics and baseline observations were extracted. The presence of structural cardiac anomalies was determined through analysis of MRI and echocardiogram imaging, in addition to review of records relating to previous operative intervention.

Results Medical records for 46 patients with Turner syndrome were reviewed, 7 (15.2\%) of whom were mosaic for $\mathrm{X}$-chromosomal monosomy. Median age was 28 years (range 21-73 years). 19/46 (41.3\%) patients had no reported structural cardiovascular anomaly. Of the 27 patients with structural anomalies, $19(70.4 \%)$ were reported to feature a bicuspid aorta, $13(28.3 \%)$ coarctation of the aorta and $10(21.7 \%)$ dilated aortic root. 13/46 (28.3\%) patients underwent surgical intervention, including 11 (23.9\%) who underwent repair of aortic coarctation.

Conclusions The presence of cardiovascular abnormalities amongst our population was lower than that reported within the literature. Patients with Turner syndrome are nevertheless likely to undergo surgical intervention. Risk stratification tools are therefore required to optimise use of resources in patients with Turner syndrome requiring routine screening for structural cardiac anomalies.

Conflicts of interest The authors declare that they have no conflicts of interest relating to this article.

\section{RELATIONSHIP OF MYOCARDIAL STRAIN AND MARKERS OF MYOCARDIAL INJURY TO PREDICT SEGMENTAL RECOVERY FOLLOWING ACUTE ST-SEGMENT ELEVATION MYOCARDIAL INFARCTION}

${ }^{1} \mathrm{JN}$ Khan*, ${ }^{2} \mathrm{JP}$ Greenwood, 'SA Nazir, ${ }^{1} \mathrm{~A}$ Singh, ${ }^{3} \mathrm{C}$ Peebles, ${ }^{4} \mathrm{~J}$ Wong, ${ }^{1} \mathrm{AH}$ Gershlick, ${ }^{1}$ GP McCann. 'Department of Cardiovascular Sciences, University of Leicester and the NIHR Leicester Cardiovascular Biomedical Research Unit, University Hospitals of Leicester NHS Trust, Glenfield Hospital, Leicester, LE3 9QP, UK; ${ }^{2}$ Multidisciplinary Cardiovascular Research Centre and the Division of Cardiovascular and Diabetes Research, Leeds Institute of Cardiovascular and Metabolic Medicine (LICAMM), University of Leeds, Leeds, LS2 9JT, UK; ${ }^{3}$ University Hospital Southampton NHS Foundation Trust and University of Southampton, Southampton SO16 6YD, UK; ${ }^{4}$ Royal Brompton and Harefield Foundation Trust, Harefield Hospital, Hill End Road, Middlesex UB9 6JH, UK

\subsection{6/heartjnl-2015-307845.18}

Background Late Gadolinium Enhancement (LGE) predicts functional recovery in stunned myocardium. Acutely post STEMI, LGE overestimates infarct and underestimates potential for functional recovery. There are no large studies comparing CMR predictors of segmental recovery in acute STEMI.

Purpose Determine whether segmental circumferential strain (Ecc), myocardial salvage (MSI), microvascular obstruction (MVO) and intramyocardial haemorrhage (IMH) predict segmental functional recovery and offer incremental predictive value to segmental extent of enhancement (SEE) acutely postPPCI.

Methods 1.5T CMR was performed in 164 patients (2624 segments) at $48 \mathrm{hr}$ and 9 month post-STEMI. LV function was assessed on wall-motion scoring on SSFP cines. Segmental dysfunction was WMS $\geq 2$, improvement was WMS decrease of $\geq 1$ and normalisation where WMS returned to 1 at follow-up. SEE and MVO were assessed on LGE using full-width half-maximum thresholding. Oedema and IMH were assessed on T2w imaging using Otsu's Automated Method. Ecc was Feature Trackingderived. MSI was the proportion of non-infarcted segmental oedema. Accuracy of baseline SEE, segmental Ecc, MSI, MVO and $\mathrm{IMH}$ in predicting improvement and normalisation in dysfunctional segments was assessed with Receiver Operator Curves.

Results 32\% of segments were dysfunctional at baseline and $19 \%$ at follow-up. With increasing SEE, segmental function worsened and proportion of dysfunctional segments recovering decreased. However $33 \%$ of SEE $>75 \%$ segments improved (Figure 1a). SEE was a strong predictor of improvement (AUC 0.708 ) and normalisation (AUC 0.807). SEE was a stronger predictor than MVO, IMH and Ecc ( $p<0.01$ for all). MVO, IMH and Ecc were weak predictors. Combining SEE with MVO, 




Abstract 18 Figure 1 (a) Recovery in dysfunctional segments at follow-up CMR by SEE. (b) ROC curve of single and combined predictors of segmental improvement in dysfunctional segments. (c) ROC curve of single and combined predictors of segmental normalisation in dysfunctional segments.

IMH, Ecc or MSI did not improve predictive accuracy versus SEE alone (Figure $1 \mathrm{~b}-1 \mathrm{c})$.

Conclusions This is the largest study assessing CMR predictors of segmental recovery in acute STEMI. Baseline SEE was the strongest predictor. Ecc, MSI, MVO and IMH provided no incremental predictive value to SEE. Functional improvement can occur where SEE $>75 \%$.

\section{THE RANDOMISED COMPLETE VS. LESION ONLY PRIMARY PCI TRIAL - CARDIOVASCULAR MRI SUBSTUDY (CVLPRIT-CMR)}

${ }^{1} \mathrm{JN}$ Khan*, ${ }^{2} \mathrm{JP}$ Greenwood, ${ }^{1} \mathrm{SA}$ Nazir, ${ }^{3} \mathrm{M}$ Dalby, ${ }^{4} \mathrm{~N}$ Curzen, ${ }^{5} \mathrm{~S}$ Hetherington, ${ }^{6} \mathrm{DJ}$ Kelly, ${ }^{2} \mathrm{D}$ Blackman, ${ }^{7} \mathrm{~A}$ Ring, ${ }^{4} \mathrm{C}$ Peebles, ${ }^{3} \mathrm{~J}$ Wong, ${ }^{8} \mathrm{M}$ Flather, ${ }^{9} \mathrm{H}$ Swanton, ${ }^{1} \mathrm{AH}$ Gershlick, ${ }^{1}$ GP McCann. 'Department of Cardiovascular Sciences, University of Leicester and the NIHR Leicester Cardiovascular Biomedical Research Unit, University Hospitals of Leicester NHS Trust, Glenfield Hospital, Leicester, LE3 9QP, UK; ${ }^{2}$ Multidisciplinary Cardiovascular Research Centre and the Division of Cardiovascular and Diabetes Research, Leeds Institute of Cardiovascular and Metabolic Medicine (LICAMM), University of Leeds, Leeds, LS2 9JT, UK; ${ }^{3}$ Royal Brompton and Harefield Foundation Trust, Harefield Hospital, Hill End Road, Middlesex UB9 6JH, UK; ${ }^{4}$ University Hospital Southampton NHS Foundation Trust and University of Southampton, Southampton SO16 6YD, UK; ${ }^{5}$ Kettering General Hospital, Rothwell Road, Kettering, NN16 8UZ, UK; ${ }^{6}$ Royal Derby Hospital, Derby DE22 3NE, UK; ' ${ }^{7}$ Leicester Clinical Trials Unit, University of Leicester, Leicester UK and Department of Mathematical Statistics and Actuarial Science, University of the Free State, PO Box 339, Bloemfontein 9300, South Africa; ${ }^{8}$ Norfolk and Norwich University Hospitals NHS Foundation Trust and Norwich Medical School, University of East Anglia Norwich NR4 7TJ, UK; ${ }^{9}$ The Heart Hospital, University College London Hospitals, 16-18 Westmoreland Street, London W1G 8PH, UK

\subsection{6/heartinl-2015-307845.19}

Background Multivessel disease (MVD) occurs in $\sim 40 \%$ of STEMI. Management is controversial. PRAMI and CVLPRIT showed improved clinical outcomes with complete versus infarct-related artery (IRA)-only revascularisation at primary percutaneous coronary intervention (PPCI). However, non-IRA PCI may cause additional infarcts. We aimed to determine whether in-hospital complete revascularisation was associated with increased myocardial injury versus an IRA-only strategy.

Methods Multicentre, prospective, randomised, blinded endpoint trial. STEMI patients with MVD and $<12 \mathrm{hr}$ symptoms were randomised to IRA-only or complete in-hospital PCI. 1.5T CMR was performed acutely (median 3 days post-PPCI) and with adenosine stress at 9 months. The primary CMR endpoint was acute infarct size on late gadolinium imaging. Myocardial salvage index (MSI) was the proportion of non-infarcted area-atrisk. $\mathrm{n}=100$ per group gave $80 \%$ power to detect $\pm 4 \%$ infarct size. The primary clinical outcome was 12 month combined MACE (death, repeat revascularisation, heart failure, MI).

Validation studies optimised infarct, area-at-risk and strain quantification. Full-width half-maximum infarct quantification was more accurate, reproducible and correlated strongest with ejection fraction (LVEF) and infarct characteristics. Otsu's Automated Thresholding most accurately and reproducibly assessed area-at-risk. Compared with tagging, Feature Tracking strain measurement was more robust, quicker, had better interobserver variability and correlated stronger with infarct, area-at-risk and MSI.

Results (summarised in Table 1) 203 patients (98 complete revascularisation, 105 IRA-only) completed acute CMR. The groups were well matched. There was no difference in infarct size, MSI, LVEF, circumferential strain or ischaemic burden between groups. Complete revascularisation patients had increased non-IRA MI at acute CMR (Figure 1). 12 month MACE was reduced in complete revascularisation patients (8.2\% vs. $17.1 \%, \mathrm{p}=0.055$, hazard ratio 0.43$)$.

Conclusions Complete revascularisation in STEMI with MVD leads to a small increase in CMR-detected non-IRA MI, but total infarct size and 12 month MACE are not increased. This 